\title{
Application of a Cloud Model-Set Pair Analysis in Efficacy Assessment for Diabetic Ulcers
}

\author{
Le Kuai, ${ }^{1,2}$ Jia-qi Xing, ${ }^{3}$ Jing-ting Zhang, ${ }^{4}$ Xun-zhe Xu, ${ }^{1,2}$ Min-feng Wu, ${ }^{1,2}$ Ke-qin Zhao, \\ Bin $\mathrm{Li}\left(\mathbb{D},{ }^{1,2,6}\right.$ and $\mathrm{Fu}-\mathrm{lun} \mathrm{Li} \mathbb{( i D}^{1,2}$ \\ ${ }^{1}$ Department of Dermatology of Yueyang Hospital, Shanghai University of TCM, Shanghai 200437, China \\ ${ }^{2}$ Institute of Dermatology, Shanghai Traditional Chinese Medicine Research Institute, Shanghai 201203, China \\ ${ }^{3}$ Acupuncture and Manipulation College, Shanghai University of Traditional Chinese Medicine, Shanghai 201203, China \\ ${ }^{4}$ Shanghai Chinese and Western Medicine Hospital, Shanghai University of TCM, Shanghai 200082, China \\ ${ }^{5}$ Zhuji Research Institute of Connection Mathematics, Zhuji 311811, China \\ ${ }^{6}$ Department of Dermatology, Shaanxi Traditional Chinese Medicine Hospital, Xian 710003, China
}

Correspondence should be addressed to Bin Li; 18930568129@163.com and Fu-lun Li; drlifulun@163.com

Received 28 October 2018; Revised 28 February 2019; Accepted 7 June 2019; Published 18 June 2019

Academic Editor: Arthur De Sá Ferreira

Copyright (C) 2019 Le Kuai et al. This is an open access article distributed under the Creative Commons Attribution License, which permits unrestricted use, distribution, and reproduction in any medium, provided the original work is properly cited.

Because treatment of diabetic ulcers includes various uncertainties, efficacy assessments are needed and significant. In previous studies, set pair analysis (SPA) has been applied to the efficacy assessments of traditional Chinese medicine (TCM) that pick out uncertainties related to the development and prognosis of disease. Optimized clinical protocols of SPA improve clinical efficacy. In the article, cloud model (CM) is employed to improve SPA, and a novel efficacy assessment method for a treatment of diabetic ulcers is proposed based on the cloud model-set pair analysis (CM-SPA). It is recommended to replace connection degree (CD) with cloud connection degree (CCD) that the efficacy assessment results are shown as normal clouds. Then, three diabetic ulcers patients treated with TCM made importance assessment by both CM-SPA and AHP based SPA. The comparison of assessment results shows that the CM-SPA is efficacious for the efficacy assessment of a treatment for diabetic ulcers and the results will be more scientific and accurate via CM-SPA.

\section{Introduction}

The refractory diabetic ulcer has confused patients for several years, especially in some developing countries. Studies show that $85 \%$ of patients suffer amputation [1]. Diabetic wound repair process is an extremely complex mechanism of signaling network which involves multiple factors such as tissues, cells, molecules, and genes. To cure the disease, many treatments were found and it is necessary to filter the best of them. Based on clinical validation, traditional Chinese medicine (TCM) has proved to be safe and curative effect in relieving pains of diabetic ulcers in recent years; furthermore, basic experiments have also proved part of mechanism on TCM in the treatment of diabetic ulcers [2-4]. However, it is hard to evaluate the exact efficacy because there are many uncertainties while healing. Efficacy assessment is widely used in the clinical evaluation. SPA could analyze fuzzy and uncertain factors which are integrated with TCM syndrome differentiation. In prophase researches, SPA is a powerful tool which could be used in systematic efficacy assessment for diabetic ulcers and improve clinical efficacy $[5,6]$. The core of SPA is to ensure the weight of each assessment unit which needs to be further improved. Index weight could be valued in several ways for different purposes. At present, there are many methods that can be used to evaluate the curative effect of diabetic ulcer, such as risk index method and analytic hierarchy process (AHP). Fuzzy methods with AHP can help index weights more solid. Index weights calculated using the above methods will greatly influence the SPA results to evaluate efficacy assessment. Index weight is a subjective conclusion with multidirectional deviation because it is a reflection confirmed via professional decision. For that reason another way is introduced to calculate the uncertainty coefficient to make several decisions unify called 
cloud model (CM). CM is composed of droplets, and droplets arrange in disorder. A droplet is a qualitative unit represented in numerical value. Single droplet is of little importance, but more cloud droplets can reflect overall characteristics of qualitative concept. CM not only reflects the uncertainty of concepts in natural language, but also reflects the relationship between randomness and fuzziness, which constitutes a qualitative and quantitative mapping. It has been widely applied in various fields. Accelerated life test data [7], stability evaluation of rock slopes [8], topology optimization [9], risk assessment [10], image segmentation [11], and other fuzzy data analysis also require CM. Moreover, CM is advanced in process ambiguous linguistic data $[12,13]$.

Integrate above means expert judgment firstly input as the linguistic data to establish CM to confirm more scientific and reasonable index weight. The study is going to prove that basing cloud model-set pair analysis (CM-SPA), which is a more valid novel efficacy assessment method. In contrast to past studies, the weight calculated by the advanced method denoted as the cloud descriptors instead of constant values. In the next step, cloud weight further computes cloud connectivity (CCD) instead of SPA connectivity (CD). Finally, efficacy assessment of three diabetic ulcers patients after TCM treatment, respectively, made by CM-SPA and AHP based SPA are showed.

\section{Method}

2.1. SPA. SPA is one of the contact mathematics methods proposed by Zhao [14], which can integrate the certainty and uncertainty of problems into unified conclusions. SPA establishes the relationship mathematical model of certainty and uncertainty, and the value of N, S, P, F meets the equation $\mathrm{N}=\mathrm{S}+\mathrm{P}+\mathrm{F}$. The connection of pair set $\mathrm{H}$ is described as $\mathrm{CD}$; an arithmetic expression for calculating the connection degree is as follows:

$$
\varphi(H)=\frac{S}{N}+\frac{F}{N} i+\frac{P}{N} j=a+b i+c j
$$

Equation (1) establishes the basic SPA model. If ' $i$ ' and ' $j$ are certainly valued, the CD could be calculated. Theoretically, we divide the discrepancy set into ' $m$ ' sets and the pattern should be expanded to general types named $m$ element model. The calculation formula is as follows:

$$
\varphi=a+\sum_{n=1}^{m-2} b_{n} i_{n}+c j
$$

In (2) ' $b_{n}$ ' denotes the discrepancy degree of ' $m$ ' sets and ' $i_{n}$ ' denotes the discrepancy coefficient of each grade.

Assume index $\mathrm{k}$ is any of indices. For the CD of index $\mathrm{k}$, value of coherence degree, distinction degree, and contrast degree is set as ' 1 'or ' 0 ', depending on its assessment value vest in effective grade or not. Usually, the main emphasis of SPA is how to ensure the index weight. In the ordinary way, shortcut process of constant was used for the index weight. As a result, the total $\mathrm{CD}$ could be calculated by

$$
\varphi=\sum_{\mathrm{k}=1}^{\mathrm{r}} \varphi_{\mathrm{k}} \omega_{\mathrm{k}}
$$

When SPA is applied in efficacy assessment, the results are subordinated to the principle of maximal connection degree, which means the maximal CD represents the CD of total indices. For a sample, assume $d=\max \left\{a, b 1, b 2 \ldots b_{k-2}\right.$, c) $(k \geq 3)$. Total CD $\varphi=a+b_{1} i_{1}+b_{2} i_{2}+\ldots+b_{k-2} i_{k-2}+c j$. Then through operation above, the efficacy grade should be $\mathrm{d}$.

In this research, we set the SPA as a 5-element pattern; then, the general form of CD was $\varphi=a+b_{1} i_{1}+b_{2} i_{2}+b_{3} i_{3}+c j$, naming the 5 corresponding uncertain factors as unimportance (I), low importance (II), medium importance (III), high importance (IV), and higher importance (V). The certainty degree of the concept can be as connection degree; then SPA can be used to aggregate of quantitative with certain degree [15].

2.2. Cloud Model. Actually, in SPA method, each weight of indexes differs from each other in the efficacy assessment. The cloud model (CM) can embody uncertainly of the concept which is composed of cloud droplets that are random values and its degree can express the fuzziness; moreover, it could be more effective in achieving the efficacy assessment [15]. Cloud model was applied to analyze the weight of index used in the SPA. Conduct an efficacy assessment of diabetic ulcers by using CM-SPA.

Assume the given universe of discourse named $\mathrm{U}$, and $\mathrm{x}$ represents any of elements $\in \mathrm{U}$. If $\mathrm{x}$ fulfill the concept $\mathrm{C}$, which is the correspondence between $\mathrm{U}$ and $\mathrm{C}(\mathrm{x}) \in[0,1]$, ' $\mathrm{x}$ ' seems to be a stochastic event with stable trends; therefore, distribution of $\mathrm{x}$ in $\mathrm{U}$ is a cloud, and each $\mathrm{x}$ is called a cloud drop.

$$
\begin{gathered}
\mu: \mathrm{U} \longrightarrow[0,1] \\
\forall \mathrm{x} \in \mathrm{U} \\
\mathrm{x} \longrightarrow \mu(\mathrm{x})
\end{gathered}
$$

Concept $\mathrm{C}=(\mathrm{Ex}, \mathrm{En}, \mathrm{He})$ is clearly defined to characterize the uncertainty in CM. Among these concepts, expected value, entropy, and hyper entropy are described as Ex, En, and He, respectively. Ex denotes the most representative of this qualitative statement in the number of domain space which reflects the position of the center of gravity. En reveals the relevance of ambiguity and randomness. He is an uncertainty measure, the entropy of entropy, which reflects the small degree of certainty of cohesion of all points representing the linguistic value in the domain interrogation; that is, the degree of aggregation of cloud droplets. $\mathrm{C}=(\mathrm{Ex}, \mathrm{En}, \mathrm{He})$ is the qualitative concept in $\mathrm{U}$ which is the quantitative universe of discourse. Assume that a measurable value $\mathrm{x}(\forall \mathrm{x} \in \mathrm{U})$ is random for $\mathrm{C}$ and $\mathrm{x}$ fulfills $\mathrm{x} \sim \mathrm{N}\left(\mathrm{Ex}, \mathrm{En}^{\prime 2}\right)$. In the same, $\mathrm{En}^{\prime}$ is also a stochastic instantiation and fulfills $\mathrm{EN} \sim \mathrm{N}\left(\mathrm{En}, \mathrm{He}^{2}\right)$. Ex satisfies the following $[8,16]$.

$$
\mu=\mathrm{e}^{(\mathrm{x}-\mathrm{Ex})^{2} / 2\left(\mathrm{En}^{\prime}\right)^{2}}
$$

Set Ex, En, and He as 5, 1, and 0.1, respectively, to assume the normal cloud which is operated by the forward cloud generator (Figure 1). Regarding the cloud drops that contribute 




Figure 1: An example of normal cloud. Assume a normal cloud and let Ex, En, and He be 5, 1, and 0.1, respectively. Meanwhile, the amounts of cloud drops are set as 1000 . Normal cloud images can be used as a reference standard for subsequent images.

to the qualitative concepts, they are mainly focused on [Ex$3 \mathrm{En}, \mathrm{Ex}+3 \mathrm{En}$ ]. Contribution of cloud drops outside [Ex-3En, $\mathrm{Ex}+3 \mathrm{En}]$ can be ignored. The definition is called the ' $3 \mathrm{En}$ rule' [16].

In order to represent the concept in $\mathrm{CM}$, the confusion degree is introduced (see (6)). It needs to be pointed out that if the confusion value is greater than or equal to (but not less than) 1 , then the cloud will be 'fog', which means concepts need to be given again.

$$
\text { confusion degree }=\frac{3 \mathrm{He}}{\mathrm{En}}
$$

There is a multitude of influencing factors of diabetic ulcer. Experts on the different symptoms of the disease about the influence mechanism of the process of generation and development have not yet been fully revealed, and the problem of disease assessment has both fuzziness and randomness. By introducing the cloud theory to study the relationship between fuzziness and randomness, the problem of quantitative evaluation of diabetic ulcer can be well solved.

2.3. CM-SPA. In the article, CM was introduced into SPA to improve methods to calculate index weight, which is defined as the cloud weight. In traditional methods, input values of SPA are linguistic variables summed up from experts. However, how to reflect the uncertainty of linguistic variables is still an issue for the great influence of efficacy assessment results. A solution is cloud weight, which replaces the traditional weight for indices, and the cloud descriptors, which represent the efficacy assessment result. Detailed steps are shown below.

(1) Each index is evaluated by the linguistic variables. Evaluation criteria are shown in Table 1; then normalized results are shown below.

$$
\mathrm{X}_{\mathrm{kl}}=\frac{\mathrm{x}_{\mathrm{kl}}}{\sum_{\mathrm{k}=1}^{\mathrm{r}} \mathrm{x}_{\mathrm{kl}}}
$$

TABLE 1: Judgment criterion of experts for index weight. The table shows the expert judgment criteria of index weight. The important level is divided into five levels from very unimportant to very important, and the corresponding weight number is gradually increased from 0 to 10 . Experts rate the importance of different symptoms.

\begin{tabular}{lc}
\hline Linguistic Variables Level & Value Range \\
\hline Very important & $(8,10]$ \\
Important & $(6,8]$ \\
Middle important & $(4,6]$ \\
Unimportant & $(2,4]$ \\
Very unimportant & $(0,2]$ \\
\hline
\end{tabular}

In (7), $\mathrm{x}_{\mathrm{kl}}$ represents the evaluations of index $\mathrm{k}$ made by expert 1 and $\mathrm{r}$ is amounts of indices, so that $\mathrm{X}_{\mathrm{kl}}$ represents the normalized value of $\mathrm{x}_{\mathrm{kl}}$.

(2) By obtaining the evaluations for index weight, the qualitative linguistic variables are transformed into quantitative values, expressed by the cloud descriptors Ex, En, and $\mathrm{He}$ (see (8)). Then, the cloud weight of index $\mathrm{k}, \omega(\mathrm{C}) \mathrm{k}=\left(\mathrm{Ex}_{\mathrm{k}}\right.$, $\left.\mathrm{En}_{\mathrm{k}}, \mathrm{He}_{\mathrm{k}}\right)$ is calculated below.

$$
\begin{aligned}
& \mathrm{Ex}_{\mathrm{k}}=\frac{1}{\mathrm{~N}} \sum_{\mathrm{l}=1}^{\mathrm{N}} \mathrm{X}_{\mathrm{kl}} \\
& \mathrm{En}_{\mathrm{k}}=\sqrt{\frac{\pi}{2} \times \frac{1}{\mathrm{~N}} \sum_{\mathrm{l}=1}^{\mathrm{N}}\left|\mathrm{X}_{\mathrm{kl}}-\mathrm{Ex}_{\mathrm{k}}\right|} \\
& \mathrm{He}_{\mathrm{k}}=\sqrt{\left|\frac{1}{\mathrm{~N}} \sum_{\mathrm{l}=1}^{\mathrm{N}}\left(\mathrm{X}_{\mathrm{kl}}-\mathrm{Ex}_{\mathrm{k}}\right)^{2}-\left(\mathrm{En}_{\mathrm{k}}\right)^{2}\right|}
\end{aligned}
$$

(3) As the cloud weight of the index was given, CCD, the total CD based on CM, can be calculated in the same way mentioned in SPA by (2) and (3) [17].

$$
\begin{aligned}
\mathrm{Ex}_{\mathrm{s}} & =\mathrm{Ex}_{1} \oplus \mathrm{Ex}_{2} \oplus \ldots \oplus \mathrm{Ex}_{\mathrm{k}}=\mathrm{Ex}_{1}+\mathrm{Ex}_{2}+\ldots \mathrm{Ex}_{\mathrm{k}} \\
\mathrm{En}_{\mathrm{s}} & =\mathrm{En}_{1} \oplus \mathrm{En}_{2} \oplus \ldots \oplus \mathrm{En}_{\mathrm{k}} \\
& =\sqrt{\mathrm{En}_{1}^{2}+\mathrm{En}_{2}^{2}+\ldots \mathrm{En}_{\mathrm{k}}^{2}} \\
\mathrm{He}_{\mathrm{s}} & =\mathrm{He}_{1} \oplus \mathrm{He}_{2} \oplus \ldots \oplus \mathrm{He}_{\mathrm{k}} \\
& =\sqrt{\mathrm{He}_{1}^{2}+\mathrm{He}_{2}^{2}+\ldots \mathrm{He}_{\mathrm{k}}^{2}}
\end{aligned}
$$

(4) Final efficacy assessment results are shown in (9) which is made up of several normal clouds. In general, if the SPA model contains several elements, there will be the same number normal clouds shown in the final efficacy assessment results. The uncertain factors assessment result is confirmed according to the '3En rule' and the maximal connection degree principle as previously mentioned.

\section{Case Study}

In this study, the key factors of diabetic ulcer were taken as the background. According to previous clinical observation, we 
TABLE 2: Introduction of the experts. The table provides an introduction to the expert, consisting of 2 students, 3 doctors-in-charge, 4 associate professors, and 3 professors, with work experience ranging from 2 to 40 years.

\begin{tabular}{lccc}
\hline & Professional position & Education background & Experience(years) \\
\hline Expert1 & Student & Bachelor & 2 \\
\hline Expert2 & Student & Master & 4 \\
\hline Expert3 & Doctor-in-charge & Master & 10 \\
\hline Expert4 & Doctor-in-charge & Master & 10 \\
\hline Expert5 & Doctor-in-charge & PhD & 15 \\
\hline Expert6 & Associate Professor & Master & 22 \\
\hline Expert7 & Associate Professor & PhD & 25 \\
\hline Expert8 & Professor & PhD & 29 \\
\hline Expert9 & Professor & PhD & 35 \\
\hline Expert10 & Professor & PhD & 40 \\
\hline
\end{tabular}

TABLE 3: Results of experts' judgments regarding the importance of assessment indices. The corresponding meanings of the $\mathrm{K}$ value are expressed as follows. K1: wound area; K2: wound depth; K3: exudates color; K4: exudates volume; K5: necrotic tissue area; K6: new granulation and epithelial tissue color; K7: new granulation and epithelial tissue area; K8: wound skin temperature; K9: wound skin color; K10: pain.

\begin{tabular}{|c|c|c|c|c|c|c|c|c|c|c|}
\hline & $\mathrm{K} 1$ & $\mathrm{~K} 2$ & K3 & $\mathrm{K} 4$ & K5 & K6 & K7 & K8 & K9 & K10 \\
\hline Expert1 & 6 & 10 & 10 & 8 & 8 & 9 & 8 & 7 & 8 & 5 \\
\hline Expert2 & 10 & 9 & 8 & 6 & 8 & 9 & 9 & 8 & 7 & 5 \\
\hline Expert3 & 7 & 10 & 6 & 5 & 8 & 9 & 8 & 5 & 5 & 5 \\
\hline Expert4 & 9 & 8 & 9 & 6 & 7 & 9 & 9 & 7 & 5 & 6 \\
\hline Expert5 & 10 & 10 & 8 & 8 & 9 & 8 & 7 & 7 & 7 & 6 \\
\hline Expert6 & 8 & 8 & 5 & 5 & 5 & 5 & 5 & 4 & 4 & 5 \\
\hline Expert7 & 6 & 7 & 6 & 6 & 5 & 7 & 8 & 8 & 7 & 7 \\
\hline Expert8 & 7 & 9 & 8 & 7 & 7 & 7 & 8 & 6 & 6 & 7 \\
\hline Expert9 & 10 & 7 & 9 & 9 & 7 & 9 & 9 & 9 & 7 & 7 \\
\hline Expert10 & 7 & 9 & 8 & 5 & 8 & 7 & 7 & 7 & 5 & 6 \\
\hline
\end{tabular}

consider the impact of diabetes ulcer prognosis indicators are wound area, wound depth, exudates color, exudates volume, necrotic tissue area, new granulation and epithelial tissue color, new granulation and epithelial tissue area, wound skin temperature, wound skin color, and pain [18]. Afterwards, preclinical studies of three patients [18] which have been approved by the Ethics Committee of Yueyang Hospital of Integrated Traditional Chinese and Western Medicine Affiliated to Shanghai University of traditional Chinese medicine (protocol 2016061) conducted an importance assessment using CM-SPA. SPA was set as a 5-element model; moreover, general type of CD was $\varphi=a+b_{1} i_{1}+b_{2} i_{2}+b_{3} i_{3}+c j$; then 5 corresponding uncertain factors were named as unimportance (I), low importance (II), medium importance (III), high importance (IV), and higher importance (V).

10 experts were invited to assess their importance based on Table 1, and their introductions were displayed in Table 2. Ex, En, and He were computed by (7) through (8). The judgment results and cloud weight of each uncertain factors are displayed in Tables 3 and 4. Based on the SPA definition that is mentioned previously, the evaluations for each importance grade were confirmed by Table 5 according to severity score for diabetic foot ulcers [19] and Table 6 is based on the patients' symptoms after the treatment with traditional Chinese medicine. The results are shown in Table 7.

\section{Results}

After obtaining the evaluations of each importance grade of the three patients, the CCD was computed by using (9), and the calculation results were shown in Table 8. Results of importance assessment analyzed by CM-SPA as shown in Figures 2, 3, and 4. In consideration of constant values of indices weights, AHP is most appropriate which is extensively used in the determination of SPA index weights. In order to validate the comparability of result, the same data was employed by AHP based SPA. The following is a brief description of the determination of indices weights obtained by AHP.

(1) Firstly, efficacy assessment for diabetic ulcers was treated as the overall goal of AHP. Then, the two basic factors for evaluating the efficacy of diabetic ulcer patients, the degree of wound healing and hypertrophic scar, were taken as the intermediate factors of analytic hierarchy process (AHP). Therefore, these ten evaluation indexes were taken as the evaluation criteria of analytic hierarchy process (AHP).

(2) Pairwise comparison matrix was used to judge both importance of criteria to middle factors and middle factors to the overall goal [16]. The matrix reflected the pairwise comparison and was compared on a scale of 1/9-9. The judgment matrix of wound healing degree and hypertrophic 
TABLE 4: Cloud weight of each index. Ex, En and He denote the expected value, entropy, and hyper entropy, respectively. Ex is the expected value of the cloud drop that can represent the qualitative concept. En reflects the dispersion degree of cloud drops, which also determines the certainty of cloud drops. He is the entropy of En. It reveals the uncertainty measurement of En that is used to settle confusion degree (see (6)). For the commonsense concept, He is smaller when the acceptance degree is higher. In the same way, He will be bigger if the concept cannot reach an agreement. After the test of (6), the distribution of Ex, En, and He in Table 4 is in accordance with the shape of cloud.

\begin{tabular}{lccc}
\hline Index & Ex & En & He \\
\hline Wound area(K1: $\left.\mathrm{cm}^{2}\right)$ & 0.11073 & 0.0217 & 0.00486 \\
Wound depth(K2: cm) & 0.12116 & 0.01886 & 0.00515 \\
Exudates color(K3) & 0.10537 & 0.01384 & 0.00412 \\
Exudates volume(K4: layers of gauze wetted) & 0.0891 & 0.01365 & 0.00451 \\
Necrotic tissue area (K5: \%) & 0.09907 & 0.01335 & 0.00348 \\
New granulation \& epithelial tissue color(K6) & 0.10844 & 0.01164 & 0.00255 \\
New granulation \& epithelial tissue area (K7:\%) & 0.10733 & 0.01166 & 0.00280 \\
Wound skin temperature(K8) & 0.09309 & 0.01464 & 0.00168 \\
Wound skin color(K9) & 0.08363 & 0.01205 & 0.00308 \\
Pain(K10:VAS) & 0.08207 & 0.01385 \\
\hline
\end{tabular}

TABLE 5: Classification of grade according to severity score for diabetic foot ulcers.

\begin{tabular}{|c|c|c|c|c|c|}
\hline \multirow{2}{*}{ Index } & \multicolumn{5}{|c|}{ Grade } \\
\hline & I & II & III & IV & $\mathrm{V}$ \\
\hline Wound area $\left(\mathrm{K} 1: \mathrm{cm}^{2}\right)$ & 0 & $1-4$ & $4-9$ & $9-16$ & $>16$ \\
\hline Wound depth(K2: $\mathrm{cm})$ & $0-1$ & $1-2$ & $2-3$ & $3-4$ & $>4$ \\
\hline Exudates color(K3) & Transparent & Red & Yellow & Green & Black \\
\hline Exudates volume(K4: layers of gauze wetted) & $0-4$ & $5-8$ & $9-12$ & $13-16$ & $>16$ \\
\hline Necrotic tissue area (K5: \%) & $0-20$ & $21-40$ & $41-60$ & $61-80$ & $81-100$ \\
\hline New granulation \& epithelial tissue color(K6) & Bright red & Red & Light red & Pink & Pale \\
\hline New granulation \& epithelial tissue area (K7:\%) & $81-100$ & $61-80$ & $41-60$ & $21-40$ & $0-20$ \\
\hline Wound skin temperature(K8) & Normal & Slightly hot & Hot & Pretty hot & Scorching hot \\
\hline Wound skin color(K9) & Normal & Reddish & Red & Bright red & Dark red \\
\hline Pain(K10:VAS) & $0-2$ & $3-4$ & $5-6$ & $7-8$ & $9-10$ \\
\hline
\end{tabular}

scar to the overall target was expressed as matrix M1 (see (10)), and the judgment matrix of wound healing degree and 10 evaluation indexes of hypertrophic scar were expressed by matrices M2 and M3 (see (11)-(12)), respectively. P1 and p2 indicated the degree of wound healing and hypertrophic scar, respectively, and $\alpha$ denoted the pairwise comparison result. The pairwise comparison results in the matrix summarized from wound-based severity score for diabetic foot ulcers [19].

$$
\begin{aligned}
M_{1} & =\left[\begin{array}{llll}
\alpha_{p 1 p 1} & \alpha_{p 1 p 2} \\
\alpha_{p 2 p 1} & \alpha_{p 2 p 2}
\end{array}\right]=\left[\begin{array}{cc}
1 & 3 \\
1 & 1
\end{array}\right] \\
M_{2} & =\left[\begin{array}{llllllllll}
\alpha_{k 1 k 1} & \alpha_{k 1 k 2} & \alpha_{k 1 k 3} & \alpha_{k 1 k 4} & \alpha_{k 1 k 5} & \alpha_{k 1 k 6} & \alpha_{k 1 k 7} & \alpha_{k 1 k 8} & \alpha_{k 1 k 9} & \alpha_{k 1 k 10} \\
\alpha_{k 2 k 1} & \alpha_{k 2 k 2} & \alpha_{k 2 k 3} & \alpha_{k 2 k 4} & \alpha_{k 2 k 5} & \alpha_{k 2 k 6} & \alpha_{k 2 k 7} & \alpha_{k 2 k 8} & \alpha_{k 2 k 9} & \alpha_{k 2 k 10} \\
\alpha_{k 3 k 1} & \alpha_{k 3 k 2} & \alpha_{k 3 k 3} & \alpha_{k 3 k 4} & \alpha_{k 3 k 5} & \alpha_{k 3 k 6} & \alpha_{k 3 k 7} & \alpha_{k 3 k 8} & \alpha_{k 3 k 9} & \alpha_{k 3 k 10} \\
\alpha_{k 4 k 1} & \alpha_{k 4 k 2} & \alpha_{k 4 k 3} & \alpha_{k 4 k 4} & \alpha_{k 4 k 5} & \alpha_{k 4 k 6} & \alpha_{k 4 k 7} & \alpha_{k 4 k 8} & \alpha_{k 4 k 9} & \alpha_{k 4 k 10} \\
\alpha_{k 5 k 1} & \alpha_{k 5 k 2} & \alpha_{k 5 k 3} & \alpha_{k 5 k 4} & \alpha_{k 5 k 5} & \alpha_{k 5 k 6} & \alpha_{k 5 k 7} & \alpha_{k 5 k 8} & \alpha_{k 5 k 9} & \alpha_{k 5 k 10} \\
\alpha_{k 6 k 1} & \alpha_{k 6 k 2} & \alpha_{k 6 k 3} & \alpha_{k 6 k 4} & \alpha_{k 6 k 5} & \alpha_{k 6 k 6} & \alpha_{k 6 k 7} & \alpha_{k 6 k 8} & \alpha_{k 6 k 9} & \alpha_{k 6 k 10} \\
\alpha_{k 7 k 1} & \alpha_{k 7 k 2} & \alpha_{k 7 k 3} & \alpha_{k 7 k 4} & \alpha_{k 7 k 5} & \alpha_{k 7 k 6} & \alpha_{k 7 k 7} & \alpha_{k 7 k 8} & \alpha_{k 7 k 9} & \alpha_{k 7 k 10} \\
\alpha_{k 8 k 1} & \alpha_{k 8 k 2} & \alpha_{k 8 k 3} & \alpha_{k 8 k 4} & \alpha_{k 8 k 5} & \alpha_{k 8 k 6} & \alpha_{k 8 k 7} & \alpha_{k 8 k 8} & \alpha_{k 8 k 9} & \alpha_{k 8 k 10} \\
\alpha_{k 9 k 1} & \alpha_{k 9 k 2} & \alpha_{k 9 k 3} & \alpha_{k 9 k 4} & \alpha_{k 9 k 5} & \alpha_{k 9 k 6} & \alpha_{k 9 k 7} & \alpha_{k 9 k 8} & \alpha_{k 9 k 9} & \alpha_{k 9 k 10} \\
\alpha_{k 10 k 1} & \alpha_{k 10 k 2} & \alpha_{k 10 k 3} & \alpha_{k 10 k 4} & \alpha_{k 10 k 5} & \alpha_{k 10 k 6} & \alpha_{k 10 k 7} & \alpha_{k 10 k 8} & \alpha_{k 10 k 9} & \alpha_{k 10 k 10}
\end{array}\right]
\end{aligned}
$$




$$
\left[\begin{array}{cccccccccc}
1 & \frac{1}{3} & 1 & 3 & 3 & 1 & 1 & 3 & 5 & 5 \\
3 & 1 & 3 & 5 & 5 & 3 & 3 & 5 & 7 & 7 \\
1 & \frac{1}{3} & 1 & 3 & 3 & 1 & 1 & 3 & 5 & 5 \\
\frac{1}{3} & \frac{1}{5} & \frac{1}{3} & 1 & 1 & \frac{1}{3} & \frac{1}{3} & 1 & 3 & 3 \\
\frac{1}{3} & \frac{1}{5} & \frac{1}{3} & 1 & 1 & \frac{1}{3} & \frac{1}{3} & 1 & 3 & 3 \\
1 & \frac{1}{3} & 1 & 3 & 3 & 1 & 1 & 3 & 5 & 5 \\
1 & \frac{1}{3} & 1 & 3 & 3 & 1 & 1 & 3 & 5 & 5 \\
\frac{1}{3} & \frac{1}{5} & \frac{1}{3} & 1 & 1 & \frac{1}{3} & \frac{1}{3} & 1 & 5 & 3 \\
\frac{1}{5} & \frac{1}{7} & \frac{1}{5} & \frac{1}{3} & \frac{1}{3} & \frac{1}{5} & \frac{1}{5} & \frac{1}{5} & 1 & 1 \\
\frac{1}{5} & \frac{1}{7} & \frac{1}{5} & \frac{1}{3} & \frac{1}{3} & \frac{1}{5} & \frac{1}{5} & \frac{1}{3} & 1 & 1
\end{array}\right]
$$

$$
M_{3}=\left[\begin{array}{cccccccccc}
\alpha_{k 1 k 1} & \alpha_{k 1 k 2} & \alpha_{k 1 k 3} & \alpha_{k 1 k 4} & \alpha_{k 1 k 5} & \alpha_{k 1 k 6} & \alpha_{k 1 k 7} & \alpha_{k 1 k 8} & \alpha_{k 1 k 9} & \alpha_{k 1 k 10} \\
\alpha_{k 2 k 1} & \alpha_{k 2 k 2} & \alpha_{k 2 k 3} & \alpha_{k 2 k 4} & \alpha_{k 2 k 5} & \alpha_{k 2 k 6} & \alpha_{k 2 k 7} & \alpha_{k 2 k 8} & \alpha_{k 2 k 9} & \alpha_{k 2 k 10} \\
\alpha_{k 3 k 1} & \alpha_{k 3 k 2} & \alpha_{k 3 k 3} & \alpha_{k 3 k 4} & \alpha_{k 3 k 5} & \alpha_{k 3 k 6} & \alpha_{k 3 k 7} & \alpha_{k 3 k 8} & \alpha_{k 3 k 9} & \alpha_{k 3 k 10} \\
\alpha_{k 4 k 1} & \alpha_{k 4 k 2} & \alpha_{k 4 k 3} & \alpha_{k 4 k 4} & \alpha_{k 4 k 5} & \alpha_{k 4 k 6} & \alpha_{k 4 k 7} & \alpha_{k 4 k 8} & \alpha_{k 4 k 9} & \alpha_{k 4 k 10} \\
\alpha_{k 5 k 1} & \alpha_{k 5 k 2} & \alpha_{k 5 k 3} & \alpha_{k 5 k 4} & \alpha_{k 5 k 5} & \alpha_{k 5 k 6} & \alpha_{k 5 k 7} & \alpha_{k 5 k 8} & \alpha_{k 5 k 9} & \alpha_{k 5 k 10} \\
\alpha_{k 6 k 1} & \alpha_{k 6 k 2} & \alpha_{k 6 k 3} & \alpha_{k 6 k 4} & \alpha_{k 6 k 5} & \alpha_{k 6 k 6} & \alpha_{k 6 k 7} & \alpha_{k 6 k 8} & \alpha_{k 6 k 9} & \alpha_{k 6 k 10} \\
\alpha_{k 7 k 1} & \alpha_{k 7 k 2} & \alpha_{k 7 k 3} & \alpha_{k 7 k 4} & \alpha_{k 7 k 5} & \alpha_{k 7 k 6} & \alpha_{k 7 k 7} & \alpha_{k 7 k 8} & \alpha_{k 7 k 9} & \alpha_{k 7 k 10} \\
\alpha_{k 8 k 1} & \alpha_{k 8 k 2} & \alpha_{k 8 k 3} & \alpha_{k 8 k 4} & \alpha_{k 8 k 5} & \alpha_{k 8 k 6} & \alpha_{k 8 k 7} & \alpha_{k 8 k 8} & \alpha_{k 8 k 9} & \alpha_{k 8 k 10} \\
\alpha_{k 9 k 1} & \alpha_{k 9 k 2} & \alpha_{k 9 k 3} & \alpha_{k 9 k 4} & \alpha_{k 9 k 5} & \alpha_{k 9 k 6} & \alpha_{k 9 k 7} & \alpha_{k 9 k 8} & \alpha_{k 9 k 9} & \alpha_{k 9 k 10} \\
\alpha_{k 10 k 1} & \alpha_{k 10 k 2} & \alpha_{k 10 k 3} & \alpha_{k 10 k 4} & \alpha_{k 10 k 5} & \alpha_{k 10 k 6} & \alpha_{k 10 k 7} & \alpha_{k 10 k 8} & \alpha_{k 10 k 9} & \alpha_{k 10 k 10}
\end{array}\right]
$$

$$
=\left[\begin{array}{llllllllll}
1 & 3 & 1 & \frac{1}{3} & \frac{1}{3} & 1 & \frac{1}{3} & 1 & \frac{1}{3} & \frac{1}{3} \\
\frac{1}{3} & 1 & \frac{1}{3} & \frac{1}{7} & \frac{1}{7} & 1 & \frac{1}{3} & 1 & \frac{1}{5} & 1 \\
1 & 3 & 1 & 1 & 1 & 5 & 1 & 5 & \frac{1}{5} & 3 \\
3 & 7 & 1 & 3 & 1 & 5 & 3 & 7 & 7 & 3 \\
3 & 7 & 1 & 3 & 1 & 1 & 3 & 3 & 7 & 9 \\
1 & 1 & \frac{1}{5} & \frac{1}{5} & 1 & 1 & 1 & 1 & 1 & 1 \\
3 & 3 & 1 & \frac{1}{3} & \frac{1}{3} & 1 & 1 & \frac{1}{3} & \frac{1}{9} & 1 \\
1 & 1 & \frac{1}{5} & \frac{1}{7} & \frac{1}{3} & 1 & 3 & 1 & \frac{1}{7} & 1 \\
3 & 5 & 5 & \frac{1}{7} & \frac{1}{7} & 1 & 9 & 7 & 1 & 1 \\
3 & 1 & \frac{1}{3} & \frac{1}{3} & \frac{1}{9} & 1 & 1 & 1 & 1 & 1
\end{array}\right]
$$

(3) Finally, indices weights were computed based on the above matrices while calculation results are shown in Table 9.
After obtaining the AHP based indices weights, CDs were calculated with (3); see Tables 7 and 9. Final results of CD were shown in Table 10. According to the maximum 
TABLE 6: Index data of three diabetic ulcer patients from preclinical studies after TCM treatment.

\begin{tabular}{lccc}
\hline Index & Cheng-ming Luan & Di-he Gu & Hong-Liu \\
\hline Wound area $\left(\mathrm{K} 1: \mathrm{cm}^{2}\right)$ & 1 & 6 & 35 \\
\hline Wound depth(K2: cm) & 0.5 & Transparent & 0.3 \\
\hline Exudates color(K3) & Transparent & 7 & 0 \\
\hline Exudates volume(K4: layers of gauze wetted) & 3 & Red \\
\hline Necrotic tissue area (K5: \%) & 0 & Red & 11 \\
\hline New granulation \& epithelial tissue color(K6) & Bright red & 0 & Bright red \\
\hline New granulation \& epithelial tissue area $(\mathrm{K} 7: \%)$ & 24 & Normal \\
\hline Wound skin temperature(K8) & Normal & Bright red \\
\hline Wound skin color(K9) & Normal & 5 & Slightly hot \\
\hline Pain(K10:VAS) & 3 & Red \\
\hline
\end{tabular}

TABLE 7: Evaluations for each important grade by combining the data in Tables 5 and 6.

\begin{tabular}{|c|c|c|c|c|c|c|c|c|c|c|c|c|c|c|c|}
\hline \multicolumn{6}{|c|}{ Cheng-ming Luan } & \multicolumn{6}{|c|}{ Di-he Gu } & \multicolumn{3}{|c|}{ Hong-Liu } & \multirow[b]{2}{*}{$\mathrm{V}$} \\
\hline & I & II & III & IV & $\mathrm{V}$ & I & II & III & IV & $\mathrm{V}$ & $\mathrm{I}$ & II & III & IV & \\
\hline $\mathrm{K} 1$ & 0 & 1 & 0 & 0 & 0 & 0 & 0 & 1 & 0 & 0 & 0 & 0 & 0 & 0 & 1 \\
\hline $\mathrm{K} 2$ & 1 & 0 & 0 & 0 & 0 & 1 & 0 & 0 & 0 & 0 & 1 & 0 & 0 & 0 & 0 \\
\hline K3 & 1 & 0 & 0 & 0 & 0 & 1 & 0 & 0 & 0 & 0 & 0 & 1 & 0 & 0 & 0 \\
\hline K4 & 1 & 0 & 0 & 0 & 0 & 0 & 1 & 0 & 0 & 0 & 0 & 0 & 1 & 0 & 0 \\
\hline K5 & 1 & 0 & 0 & 0 & 0 & 1 & 0 & 0 & 0 & 0 & 1 & 0 & 0 & 0 & 0 \\
\hline K6 & 1 & 0 & 0 & 0 & 0 & 0 & 1 & 0 & 0 & 0 & 1 & 0 & 0 & 0 & 0 \\
\hline K7 & 0 & 0 & 0 & 1 & 0 & 0 & 0 & 0 & 0 & 1 & 0 & 0 & 1 & 0 & 0 \\
\hline K8 & 1 & 0 & 0 & 0 & 0 & 1 & 0 & 0 & 0 & 0 & 0 & 1 & 0 & 0 & 0 \\
\hline K9 & 1 & 0 & 0 & 0 & 0 & 0 & 0 & 0 & 1 & 0 & 0 & 0 & 1 & 0 & 0 \\
\hline K10 & 0 & 1 & 0 & 0 & 0 & 0 & 0 & 1 & 0 & 0 & 0 & 0 & 0 & 1 & 0 \\
\hline
\end{tabular}

TABLE 8: Calculation results of CCD. Ex, En, and He denote the expected value, entropy, and hyper entropy, respectively. Ex is the expected value of the cloud drop that can represent the qualitative concept. En reflects the dispersion degree of cloud drops, which also determines the certainty of cloud drops. He is the entropy of En. It reveals the uncertainty measurement of En that is used to settle confusion degree (see (6)). For the commonsense concept, He is smaller when the acceptance degree is higher. In the same way, He will be bigger if the concept cannot reach an agreement. After the test of (6), the distribution of Ex, En, and He in Table 8 is in accordance with the shape of cloud.

\begin{tabular}{lcccccccrr}
\hline & \multicolumn{3}{c}{ Cheng ming Luan } & \multicolumn{3}{c}{ Di-he Gu } & \multicolumn{3}{c}{ Hong-Liu } \\
& Ex & En & He & Ex & En & He & Ex & He \\
\hline I & 0.6999 & 0.0375 & 0.0097 & 0.4187 & 0.0307 & 0.0076 & 0.3287 & 0.0258 \\
II & 0.1928 & 0.0257 & 0.0051 & 0.1975 & 0.0179 & 0.0052 & 0.1985 & 0.0201 & 0.0045 \\
III & 0 & 0 & 0 & 0.1928 & 0.0257 & 0.0051 & 0.2801 & 0.0216 & 0.0061 \\
IV & 0.1073 & 0.0117 & 0.0028 & 0.0836 & 0.012 & 0.0031 & 0.0821 & 0.0138 & 0.0015 \\
V & 0 & 0 & 0 & 0.1073 & 0.0117 & 0.0028 & 0.1107 & 0.0217 & 0.0049 \\
\hline
\end{tabular}

connectivity principle and '3En rule', as mentioned earlier, in Figure 2, the CDs of grades I, II, and IV were mainly focused on $[0.5873,0.8124],[0.1156,0.2700]$, and $[0.0723,0.1423]$, respectively. There was no intersection between grade I and the other grades. In other words, the grade of Cheng-ming Luan completely belonged to grade I and the efficacy grade of Cheng-ming Luan was very high efficacy. In Figure 3, the CDs of grades I, II, III, IV, and V were mainly focused on $[0.3267,0.5107],[0.1437,0.2514],[0.1156,0.2700]$, $[0.0475,0.1198]$, and $[0.0723,0.1423]$, respectively. There was no intersection between grade I and the other grades. In other words, the grade of Di-he Gu completely belonged to grade I and the efficacy grade of Di-he $\mathrm{Gu}$ was very high efficacy. In Figure 4, the CDs of grades I, II, III, IV, and V were mainly focused on [0.2510,0.4063], [0.1380, 0.2589], $[0.2152,0.3449],[0.0405,0.1236]$, and $[0.0456,0.1758]$, respectively. It can be concluded that the intersection scope of grade I and grade III was the maximum; thus, the relevance of grade I to grade III was closer than for other grades. Then, it was summarized that the grade of Hong-Liu was between grade I and grade III and closer to grade I. That is to say, the efficacy grade of Hong-Liu was between very high efficacy and middle efficacy and closer to very high efficacy. 


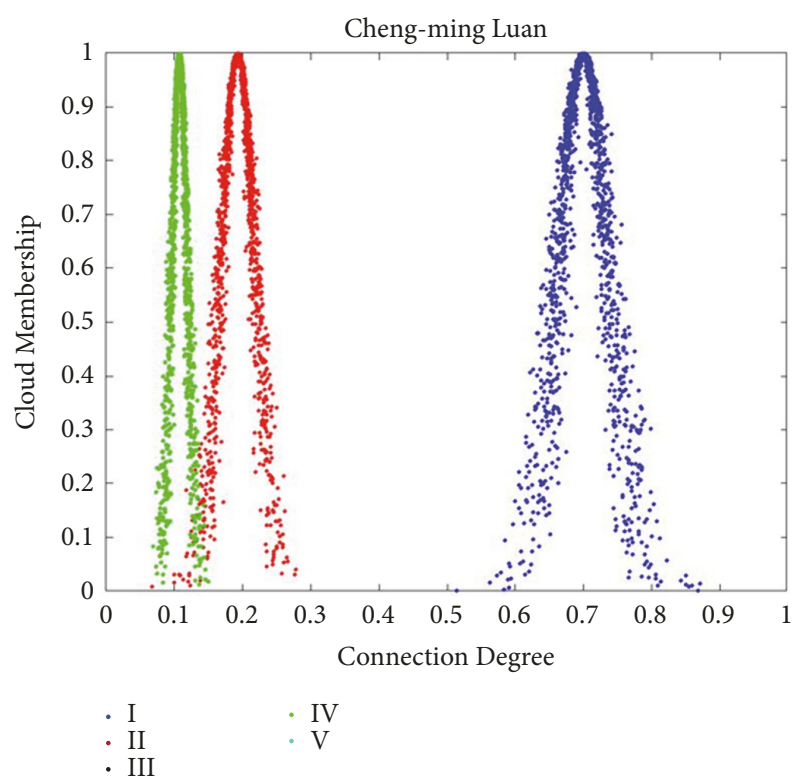

FIGURE 2: Normal clouds of CCD for Cheng-ming Luan. Suppose the number of cloud droplets is 1000; the normal cloud corresponding to each evaluation level is generated using the results of CCD operations and algorithms written on the Matlab platform, and the cloud droplets corresponding to each level are shown in the legend. According to the maximum connectivity principle and ' $3 E n$ rule', the grade of Cheng-ming Luan completely belonged to grade I and the efficacy grade of Cheng-ming Luan was very high efficacy.

In Table 10, similar results were obtained by AHP that after the treatment of traditional Chinese medicine; the curative effect ratings of the three people all belonged to grade I according to the maximum connectivity principle that also confirms the scientific nature of SPA-CM to a certain extent.

\section{Discussion}

Diabetic ulcer is a system of uncertainty such as wound area, wound depth, and exudate color [18]. At present, there are many methods that can be used to evaluate the curative effect of diabetic ulcer, such as risk index method, analytic hierarchy process (AHP), gray relational analysis, and bow tie model $[16,20]$. These evaluation methods have their own advantages and disadvantages; disadvantages are mainly for the subjectivity of weight assignment, ignoring the uncertainty of the evaluation process.

Based on the above research status, we intend to solve the subjective problem of weight assignment of uncertain factors in the evaluation system and combined with the mathematical method to study uncertain factors to carry out comprehensive evaluation of diabetic ulcer patients. At present, SPA has been used in a host of fields of efficacy assessment. For instance, SPA based layer of protection analysis has been applied in quantitative risk assessment [21]. Besides, SPA has been used to evaluate the emergency response capacity of large airports, making sure the factors and mechanism of airport vulnerability and emergency response capacity

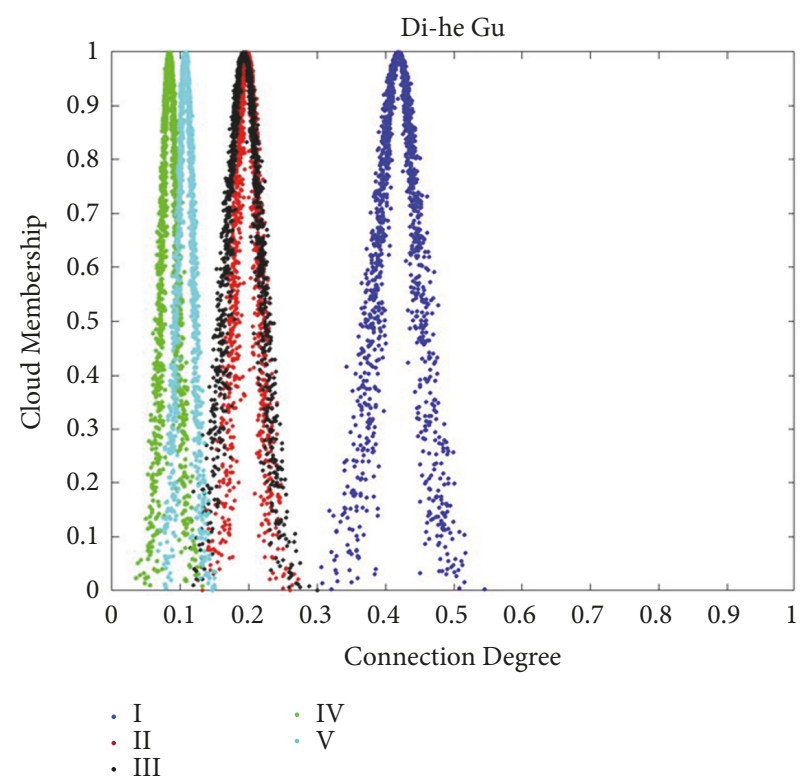

FIgURE 3: Normal clouds of CCD for Di-he Gu. Suppose the number of cloud droplets is 1000; the normal cloud corresponding to each evaluation level is generated using the results of CCD operations and algorithms written on the Matlab platform, and the cloud droplets corresponding to each level are shown in the legend. According to the maximum connectivity principle and ' $3 E$ n rule', the grade of Di-he Gu completely belonged to grade I and the efficacy grade of Cheng-ming Luan was very high efficacy.

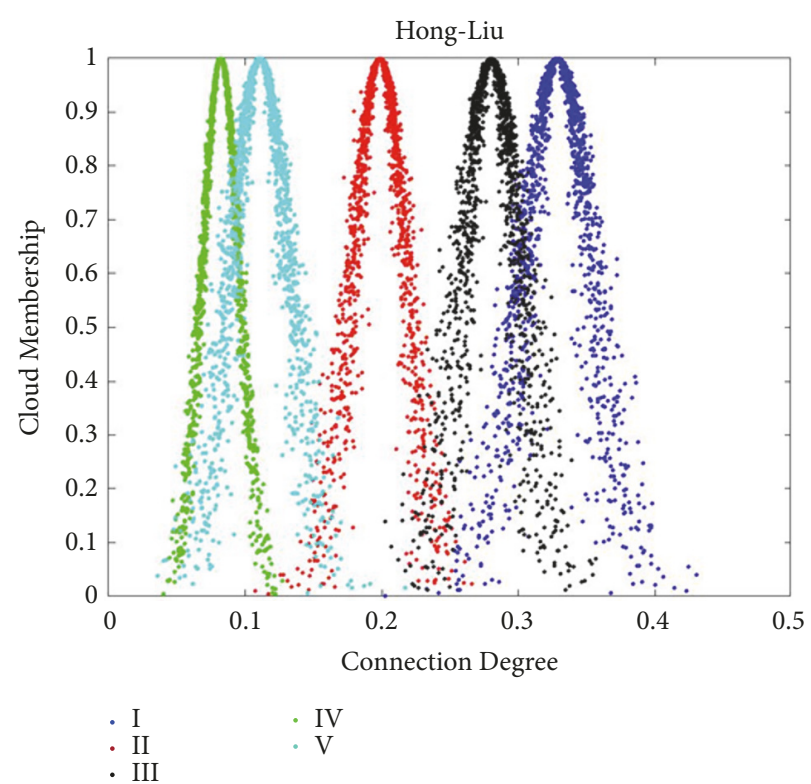

Figure 4: Normal clouds of CCD for Hong-Liu. Suppose the number of cloud droplets is 1000; the normal cloud corresponding to each evaluation level is generated using the results of CCD operations and algorithms written on the Matlab platform, and the cloud droplets corresponding to each level are shown in the legend. According to the maximum connectivity principle and '3En rule, the grade of Hong-Liu was between grade I and grade III and closer to grade I. Hence, the efficacy grade of Hong-Liu was between very high efficacy and middle efficacy and closer to very high efficacy. 
TABLE 9: Indices weights obtained by AHP. The table is the weight of ten indexes calculated according to the matrix, in which the judgment matrix of the criterion layer to the target layer and the judgment matrix of the scheme layer to the criterion layer satisfy the consistency.

\begin{tabular}{ccccccccccc}
\hline & K1 & K2 & K3 & K4 & K5 & K6 & K7 & K8 & K9 & K10 \\
\hline weight & 0.0658 & 0.0916 & 0.1163 & 0.1706 & 0.1996 & 0.0755 & 0.0716 & 0.0456 & 0.1191 & 0.0443 \\
\hline
\end{tabular}

TABLE 10: Calculation results of CD. The table is the decision set calculated based on (3) and Tables 7 and 9 . The curative effect ratings of Cheng-ming Luan, Di-he Gu and Hong-Liu all belonged to grade I according to the maximum connectivity principle.

\begin{tabular}{lccc}
\hline & Cheng-ming Luan & Di-he Gu & Hong-Liu \\
\hline I & 0.8183 & 0.4531 & 0.3667 \\
\hline II & 0.1101 & 0.2461 & 0.1619 \\
\hline III & 0.0000 & 0.1101 & 0.3614 \\
\hline IV & 0.0716 & 0.1191 & 0.0443 \\
\hline V & 0.0000 & 0.0716 & 0.0658 \\
\hline
\end{tabular}

on airport flexibility, which has improved the resilience of airports [22, 23].

In this paper, an evaluation method combining cloud model (CM) with set pair analysis (SPA) to deal with uncertain factors is adopted. This method uses the fuzziness and randomness of cloud model to determine cloud weight and reduces the subjectivity of assignment. On this basis, combined with set pair analysis, the comprehensive evaluation of diabetic ulcer patients was carried out, and the comprehensive evaluation set pair cloud model was obtained, so as to determine the curative effect rating of the evaluated object and to show it intuitively in the form of cloud droplets.

\section{Conclusions}

(1) In the paper, cloud model (CM) was used to improve set pair analysis (SPA). The cloud weight should be used in the analysis of SPA. Different from the traditional index weights of SPA as constant values, cloud weights were defined as the cloud descriptors which can reflect the fuzziness and randomness of experts' judgments. Thus the subjectivity of index weight determination can be avoided by using CMSPA.

(2) The obtained assessment results by AHP based SPA were not reasonable and precise enough due to the influences of other parameters of the CD were neglected. For example, in regard to the assessment result of Hong-Liu, though assessment values of grade I (0.3667) and grade III (0.3614) were almost equal, the assessment result can only confirmed to be grade I due to the maximal connection degree principle while CM-SPA came to the conclusion between grade I and grade III and closer to grade I. In addition, with the exception of the maximal parameter of the $\mathrm{CD}$, a slice of other parameters were not considered in confirming assessment results even though values of them were not ' 0 '. In contrast to CM-SPA, efficacy evaluation results of the classical method AHP can only be reflected by the obviously different efficacy level. For the CM-SPA, slight variation of efficacy treatments will be caught for its smaller precision, and the evaluation results will be more accurate and realistic.
(3) For the CM-SPA, further and complete assessments should be made based on the randomness and fuzziness of $\mathrm{CM}$; the assessment results will be more precise and reasonable.

\section{Data Availability}

All of the data used to support the findings of this study are available from the corresponding author upon request.

\section{Conflicts of Interest}

The authors declare that they have no conflicts of interest.

\section{Authors' Contributions}

Fu-lun Li and Bin Li conceived and designed the work. Le Kuai, Jia-qi Xing, and Jing-ting Zhang performed data analysis. Xun-zhe $\mathrm{Xu}, \mathrm{Min}$-feng $\mathrm{Wu}$, and Ke-qin Zhao interpreted results and drafted and critically evaluated the manuscript. All the authors read and approved the final manuscript.

\section{Acknowledgments}

This study was supported by the national key research and development Program of China (no. SQ2018YFC1705301), grant no. 81673866 from the National Science Foundation (NSFC) of China, the project of Shanghai Shen-kang Development (16CR2035B), Hundred Talents Program of Shaanxi (SXBR9053), Shanghai leading talent (no. 039), Shanghai Development Office of TCM [nos. ZY(2018-2020)-FWTX1008, ZY(2018-2020)-CCCX-2004-08, and ZY(2018-2020)FWTX-4010], the Shanghai Science and Technology Committee (no.16411955000), Shanghai Shuguang Project (no. 17SG41), Key Discipline Projects of Shanghai Pudong Health and Family Planning Commission (no. PWZxq2017-16), Shanghai Municipal Health and Family Planning Commission (no. ZY3-JSFC-1-1007), and Tenth Batch of College Students' Science and Technology Projects of Shanghai University of Traditional Chinese Medicine [JX61.08.97, JX61.08.98]. We thank Yue Luo for proofreading the manuscript. 


\section{Supplementary Materials}

We used Metlab software to write code to calculate the Ex, En, and He of SPA-CM by applying (8) and (9) and the weight of AHP's ten metrics. The supplementary material is the calculation code of Metlab. (Supplementary Materials)

\section{References}

[1] N. H. Cho, J. E. Shaw, S. Karuranga et al., "IDF Diabetes Atlas: Global estimates of diabetes prevalence for 2017 and projections for 2045," Diabetes Research and Clinical Practice, 2018.

[2] F.-L. Li, H. Deng, H.-W. Wang et al., "Effects of external application of chinese medicine on diabetic ulcers and the expressions of $\beta$-catenin, c-myc and K6," Chinese Journal of Integrative Medicine, vol. 17, no. 4, pp. 261-266, 2011.

[3] L. Kuai, J. T. Zhang, Y. Deng, S. Xu, and X. Z. Xu, "Shengji Hua-yu formula promotes diabetic wound healing of reepithelization via Activin/Follistatin regulation," BMC Complementary and Alternative Medicine, vol. 18, no. 1, p. 32, 2018.

[4] Y. F. Wang, X. Li, R. Xu, W. C. Jiang, F. L. Li, and K. Ze, "Effect of Shengji Huayu Recipe on the expression of MMP-3 and TIMP-1 in skin ulcer tissue of diabetic rats," Chinese Journal of Integrated Traditional and Western Medicine, vol. 34, no. 2, pp. 218-223, 2014.

[5] F. Li, K. Ze, -X. Cao, X. LI, B. LI, and J. Cheng, "and R.Xu, To establish the syndrome-typing model of psoriasis vulgaris blood-heat syndrome based on the set pair analysis method," Chinese Journal of Integrated Traditional and Western Medicine, vol. 32, no. 10, pp. 1308-1312, 2012.

[6] X. J. Hong, X. Z. Xu, Y. Ru et al., "Clinical re-research on bloodheat syndrome of psoriasis vulgaris based on set pair analysis potential results," Journal of Traditional Chinese Medicine, vol. 33, no. 3, pp. 1160-1163, 2018.

[7] W. Zhang, S. Liu, B. Sun, Y. Liu, and M. Pecht, "A cloud modelbased method for the analysis of accelerated life test data," Microelectronics Reliability, vol. 55, no. 1, pp. 123-128, 2015.

[8] Z. Liu, J. Shao, W. Xu, and F. Xu, "Comprehensive stability evaluation of rock slope using the cloud model based approach," Rock Mechanics and Rock Engineering, vol. 47, no. 6, pp. 22392252, 2013.

[9] J. Liu, G. Wen, and Y. M. Xie, "Layout optimization of continuum structures considering the probabilistic and fuzzy directional uncertainty of applied loads based on the cloud model," Structural and Multidisciplinary Optimization, vol. 53, no. 1, pp. 81-100, 2016.

[10] H. Zhao and N. Li, "Risk evaluation of a UHV power transmission construction project based on a cloud model and FCE method for ustainability," Sustainability, vol. 7, no. 3, pp. 28852914, 2015.

[11] K. Qin, K. Xu, F. Liu, and D. Li, "Image segmentation based on histogram analysis utilizing the cloud model," Computers \& Mathematics with Applications, vol. 62, no. 7, pp. 2824-2833, 2011.

[12] J. Wang, P. Lu, H. Zhang, and X. Chen, "Method of multicriteria group decision-making based on cloud aggregation operators with linguistic information," Information Sciences, vol. 274, pp. 177-191, 2014.

[13] J. Q. Wang, J. J. Peng, H. Y. Zhang, T. Liu, and X. H. Chen, "An uncertain linguistic multi-criteria group decision making method based on a cloud model," Group Decision and Negotiation, vol. 25, no. 1, pp. 171-192, 2015.
[14] K. Q. Zhao, "Theory and analysis of set pair-a new concept and system analysis method," in Conference Thesis of System Theory and Regional Planning, pp. 87-91, 1989.

[15] A. Wu, Z. Ma, and G. Zeng, "Set pair fuzzy decision method based on cloud model," Journal of Electronics, vol. 25, no. 2, pp. 215-219, 2016.

[16] F. Yan, K. Xu, and Y. Deng, "Application of a cloud modelset pair analysis in hazard assessment for biomass gasification stations," PLoS ONE, vol. 12, no. 1, Article ID e0170012, 2017.

[17] J.-Q. Wang, J.-J. Peng, H.-Y. Zhang, T. Liu, and X.-H. Chen, "An uncertain linguistic multi-criteria group decision making method based on a cloud model," Group Decision and Negotiation, vol. 1, pp. 171-192, 2015.

[18] M. F. Wu, L. Yan, D. J. Guo et al., "Microbial diversity of chronic wound and successful management of traditional chinese medicine," Evidence-Based Complementary and Alternative Medicine, vol. 2018, Article ID 9463295, 13 pages, 2018.

[19] S. Beckert, M. Witte, C. Wicke, A. Königsrainer, and S. Coerper, "A new wound-based severity score for diabetic foot ulcers: A prospective analysis of 1,000 patients," Diabetes Care, vol. 29, no. 5, pp. 988-992, 2006.

[20] F. Yan and K. Xu, "A set pair analysis based layer of protection analysis and its application in quantitative risk assessment," Journal of Loss Prevention in the Process Industries, vol. 55, pp. 313-319, 2018.

[21] Q. Xu, K. Xu, and Y. Deng, "Mine safety assessment using gray relational analysis and bow tie model," PLoS ONE, vol. 13, no. 3, Article ID e0193576, 2018.

[22] D. Bao and X. Zhang, "Measurement methods and influencing mechanisms for the resilience of large airports under emergency events," Transportmetrica A: Transport Science, vol. 14, no. 10, pp. 855-880, 2018.

[23] J. Zhang, D. Cliff, K. Xu, and G. You, "Focusing on the patterns and characteristics of extraordinarily severe gas explosion accidents in Chinese coal mines," Process Safety and Environmental Protection, vol. 117, pp. 390-398, 2018. 


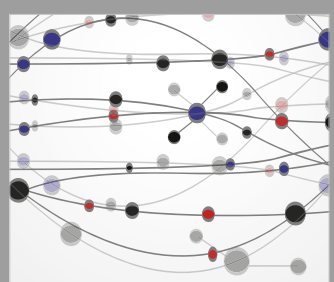

The Scientific World Journal


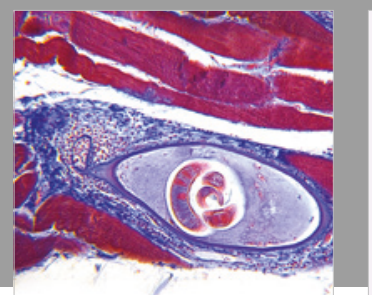

Gastroenterology Research and Practice

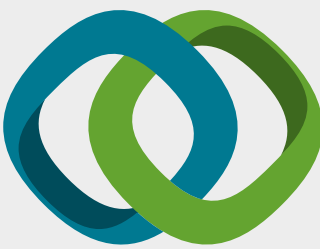

\section{Hindawi}

Submit your manuscripts at

www.hindawi.com


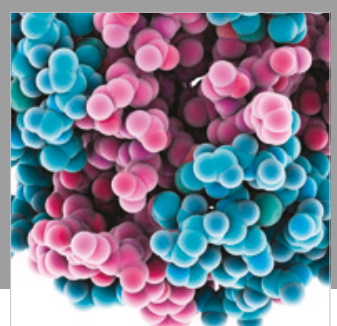

ournal of

Diabetes Research



Disease Markers
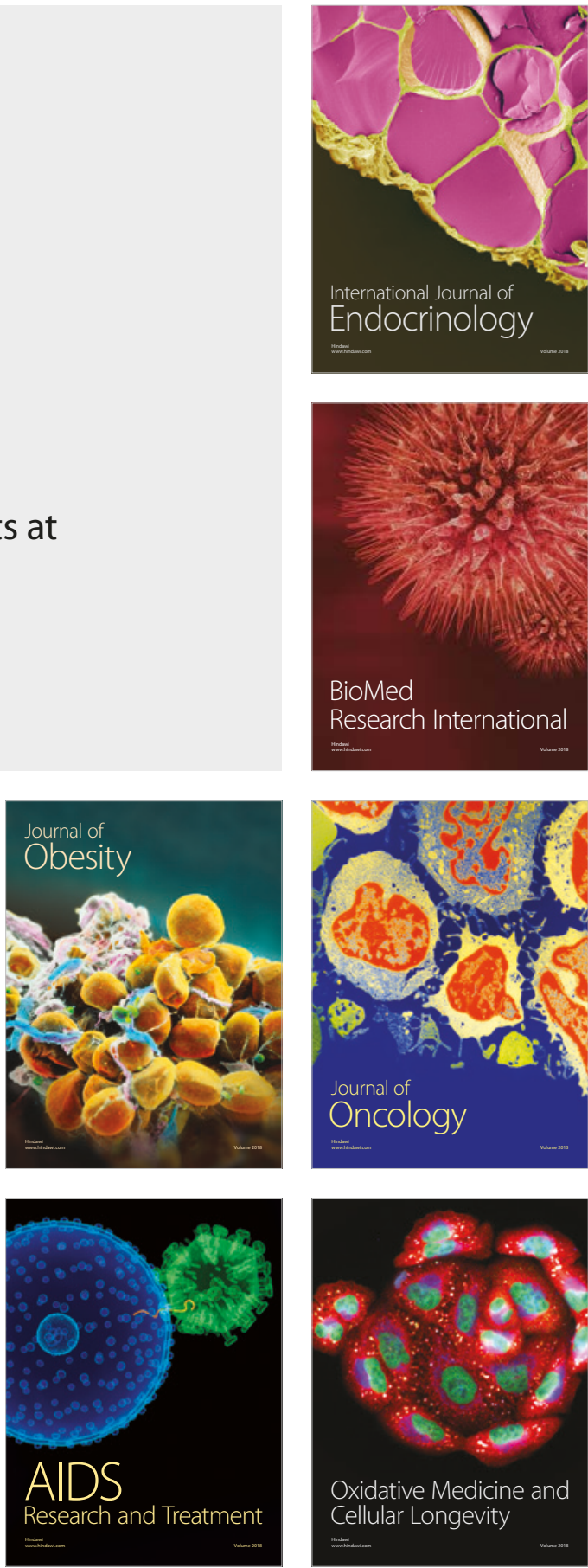